KYUNGPOOK Math. J. 49(2009), 81-93

\title{
Application of Bifurcation Method to a Generalized Modified Boussinesq Equation
}

Ming SONG*

Department of Mathematics, Faculty of Sciences, Yuxi Normal University, Yuxi, 653100, P. R. China

e-mail : songming12_15@163.com

ChengXi YANG

Department of Mathematics of Yuxi Normal Collage, Yuxi, 653100, P. R. China e-mail : ychx@yxtc.net

Abstract. Bifurcation method of dynamical systems is employed to investigate exact solitary wave solutions and kink wave solutions in the generalized modified Boussinesq equation. Under some parameter conditions, their explicit expressions are obtained. Some previous results are extended.

\section{Introduction and main results}

Zhang et al.[1] and Kaya[2] considered the following generalized modified Boussinesq(GMB) equation

$$
u_{t t}-\delta u_{t t x x}-\left(a_{1} u+a_{2} u^{p+1}+a_{3} u^{2 p+1}\right)_{x x}=0,
$$

where $\delta \neq 0, p \geqslant 1, a_{1}, a_{2}$ and $a_{3}$ are arbitrary constants. This equation is used to represent a nonlinear model of longitudinal wave propagation of elastic rods [2]. When $\delta=1, a_{1}=1, a_{2}=\frac{1}{p+1}$ and $a_{3}=0,(1.1)$ becomes the equation

$$
u_{t t}-u_{t t x x}-\left(u+\frac{1}{p+1} u^{p+1}\right)_{x x}=0,
$$

which was studied in [3], [4]. Clarkson et al.[3] and Bogolubsky[4] gave some solitary wave solutions of (1.2) with $p=1,2,4$ and studied the interaction of two solitary waves numerically. When $\delta=1, a_{1}=0, a_{2}=-\frac{1}{2}, a_{3}=0$ and $p=1,(1.1)$ reduces to the equation

$$
u_{t t}-u_{t t x x}+\frac{1}{2}\left(u^{2}\right)_{x x}=0 .
$$

\footnotetext{
* Corresponding author.
}

Received 17 September 2007; revised 1 April 2008; accepted 1 April 2008.

2000 Mathematics Subject Classification: 34A20, 34C35, 35B65, 58F05, 76B25.

Key words and phrases: bifurcation of phase portraits, solitary wave solutions, Kink wave solutions. 
Parker[5] also studied the solitary wave and exact solutions of (1.3). When $\delta=$ $1, p=1,2$, (1.1) reduces to the equation

$$
u_{t t}-u_{t t x x}-\left(a_{1} u+a_{2} u^{2}+a_{3} u^{3}\right)_{x x}=0,
$$

and

$$
u_{t t}-u_{t t x x}-\left(a_{1} u+a_{2} u^{3}+a_{3} u^{5}\right)_{x x}=0 .
$$

Zhang and $\mathrm{Ma}[6]$ gave some explicit solitary wave solutions of (1.4) and (1.5) by using the method of solving algebraic equations. Li and Zhang[7] gave many explicit solitary wave solutions and kink wave solutions of (1.4) and (1.5) by using the bifurcation method. Zhang et al.[1] used the judgement methods and solution formulae to obtain two bell profile solitary wave solutions

$$
u(x, t)=\left(\frac{\frac{\left|a_{1}-v^{2}\right|(p+2) \sqrt{p+1}}{\sqrt{(p+1) a_{2}^{2}-a_{3}\left(a_{1}-v^{2}\right)(p+2)^{2}}} \operatorname{sech}^{2} \frac{p}{2} \sqrt{-\frac{a_{1}-v^{2}}{\delta v^{2}}}\left(x-v t+\xi_{0}\right)}{2+\left(-1+\frac{a_{2}|\delta| \sqrt{p+1}}{\delta \sqrt{(p+1) a_{2}^{2}-a_{3}\left(a_{1}-v^{2}\right)(p+2)^{2}}}\right) \operatorname{sech}^{2} \frac{p}{2} \sqrt{-\frac{a_{1}-v^{2}}{\delta v^{2}}}\left(x-v t+\xi_{0}\right)}\right)^{\frac{1}{p}},
$$

$$
u(x, t)=\left(\frac{-\frac{\left|a_{1}-v^{2}\right|(p+2) \sqrt{p+1}}{\sqrt{(p+1) a_{2}^{2}-a_{3}\left(a_{1}-v^{2}\right)(p+2)^{2}}} \operatorname{sech}^{2} \frac{p}{2} \sqrt{-\frac{a_{1}-v^{2}}{\delta v^{2}}}\left(x-v t+\xi_{0}\right)}{2-\left(1+\frac{a_{2}|\delta| \sqrt{p+1}}{\delta \sqrt{(p+1) a_{2}^{2}-a_{3}\left(a_{1}-v^{2}\right)(p+2)^{2}}}\right) \operatorname{sech}^{2} \frac{p}{2} \sqrt{-\frac{a_{1}-v^{2}}{\delta v^{2}}}\left(x-v t+\xi_{0}\right)}\right)^{\frac{1}{p}}
$$

and a kink profile solitary wave solution

$$
u(x, t)=\left(-\frac{a_{2}(p+1)}{2 a_{3}(p+2)}\left[1 \pm \tanh \frac{p}{2} \sqrt{-\frac{a_{1}-v^{2}}{\delta v^{2}}}\left(x-v t+\xi_{0}\right)\right]\right)^{\frac{1}{p}}
$$

where wave velocity $v^{2}=a_{1}-\frac{a_{2}^{2}(p+1)}{a_{3}(p+2)^{2}}$, and when $p=1, a_{2}=0$, gave a kink wave solution

$$
u(x, t)= \pm \sqrt{-\frac{a_{1}-v^{2}}{a_{3}}} \tanh \frac{1}{2} \sqrt{\frac{2\left(a_{1}-v^{2}\right)}{\delta v^{2}}}\left(x-v t+\xi_{0}\right)
$$

for $(1.1)$.

Zhang[8] used the bifurcation method to obtain a solitary wave solution and two kink wave solutions of (1.1) when $p$ is even, $\delta=1, a_{3}<0, a_{2}>0$ and $\frac{a_{2}^{2}}{4 a_{3}}<a_{1}-c^{2}<0$. Kaya[2] used the Adomian decomposition method to obtain a solitary wave solution

$$
u(x, t)=S_{1}(1+\tanh R(x-c t))^{\frac{1}{p}},
$$


for (1.1), where $p=1,4$,

$$
S_{1}=\frac{a_{2}(p+1)}{2 a_{3}(p+2)}, \quad R=p \sqrt{\frac{-a_{1}^{2}+\zeta}{\zeta^{2} \delta}}, \quad \zeta=\sqrt{a_{1}-\frac{a_{2}^{2}(p+1)}{a_{3}(p+2)^{2}}} .
$$

Rafei et al.[9] used the homotopy perturbation method to obtain a approximate solitary wave solution of (1.1).

In this paper, we employ similar method used $[10,11]$ to investigate solitary wave solutions and kink wave solutions of (1.1). To facilitate discussions, we let

$$
a_{3}^{*}=\frac{(p+1) a_{2}^{2}}{(p+2)^{2}\left(a_{1}-c^{2}\right)}
$$

for given $p, a_{1}, a_{2}$ and constant $c \neq 0$. Our main results are as follows:

\subsection{Solitary wave solutions}

Under some parameter conditions, we give some solitary wave solutions of (1.1) in the following Theorem 1 and Theorem 2.

Theorem 1. (1) When $p$ is odd, $a_{2} \neq 0$ and $\delta, a_{1}, a_{3}$ satisfy one of the following conditions

(a) $\delta>0, a_{3}>0$ and $a_{1}-c^{2}<0$,

(b) $\delta<0, a_{3}<0$ and $a_{1}-c^{2}>0$,

(1.1) has two solitary wave solutions

$$
u_{1}(x, t)=\left(\frac{(p+2)\left(a_{1}-c^{2}\right)}{\alpha \cosh \beta(x-c t)-a_{2}}\right)^{\frac{1}{p}}
$$

and

$$
u_{2}(x, t)=\left(\frac{(p+2)\left(c^{2}-a_{1}\right)}{\alpha \cosh \beta(x-c t)+a_{2}}\right)^{\frac{1}{p}}
$$

where

$$
\alpha=\sqrt{\frac{(p+1) a_{2}^{2}+(p+2)^{2}\left(c^{2}-a_{1}\right) a_{3}}{p+1}}, \quad \beta=\frac{p}{|c|} \sqrt{\frac{c^{2}-a_{1}}{\delta}} .
$$

(2) When $p$ is odd, $a_{2} \neq 0$ and $\delta, a_{1}, a_{3}$ satisfy one of the following conditions

(a) $\delta>0, a_{1}-c^{2}<0$ and $a_{3}^{*}<a_{3}<0$,

(b) $\delta<0, a_{1}-c^{2}>0$ and $0<a_{3}<a_{3}^{*}$,

(1.1) has a solitary wave solution $u_{1}(x, t)$.

(3) When $p$ is odd, $\delta a_{3}>0, a_{2} \neq 0$ and $a_{1}-c^{2}=0$, (1.1) has a solitary wave solution

$$
u_{3}(x, t)=\left(\frac{4 \gamma \delta c^{2}}{\gamma^{2} p^{2}(x-c t)^{2}-4 \mu \delta c^{2}}\right)^{\frac{1}{p}}
$$


where

$$
\gamma=-\frac{2 a_{2}}{p+2}, \quad \mu=-\frac{a_{3}}{p+1} .
$$

Theorem 2. (1) When $p$ is even and $\delta, a_{1}, a_{2}, a_{3}$ satisfy one of the following conditions
(a) $\delta>0, a_{1}-c^{2}<0, a_{2} \neq 0$ and $a_{3}>0$,
(b) $\delta>0, a_{1}-c^{2}<0, a_{2}>0$ and $a_{3}^{*}<a_{3}<0$,
(c) $\delta<0, a_{1}-c^{2}>0, a_{2} \neq 0$ and $a_{3}<0$,
(d) $\delta<0, a_{1}-c^{2}>0, a_{2}<0$ and $0<a_{3}<a_{3}^{*}$,

(1.1) has two solitary wave solutions $\pm u_{2}(x, t)$.

(2) When $p$ is even, $a_{1}-c^{2}=0$ and $\delta, a_{2}, a_{3}$ satisfy one of the following conditions

(a) $\delta>0, a_{2}<0$ and $a_{3}>0$,

(b) $\delta<0, a_{2}>0$ and $a_{3}<0$,

(1.1) has two solitary wave solutions $\pm u_{3}(x, t)$.

\subsection{Kink wave solutions}

Under some parameter conditions, we give some kink wave solutions of (1.1) in the following Theorem 3 and Theorem 4.

Theorem 3. When $p$ is odd, $a_{2} \neq 0, a_{3}=a_{3}^{*}$ and $\delta\left(a_{1}-c^{2}\right)<0,(1.1)$ has two kink wave solutions

$$
u_{4}(x, t)=\left(\frac{(p+2)\left(c^{2}-a_{1}\right)}{\omega e^{-\beta(x-c t)}+a_{2}}\right)^{\frac{1}{p}}
$$

and

$$
u_{5}(x, t)=\left(\frac{(p+2)\left(c^{2}-a_{1}\right)}{\omega e^{\beta(x-c t)}+a_{2}}\right)^{\frac{1}{p}}
$$

where $\omega$ is an arbitrary constant satisfied $\omega a_{2}>0$ and $\beta$ is in (1.15).

Theorem 4. When $p$ is even, $a_{3}=a_{3}^{*}$ and $\delta, a_{1}, a_{2}$ satisfy one of the following conditions

(a) $\delta>0, a_{2}>0$ and $a_{1}-c^{2}<0$,

(b) $\delta<0, a_{2}<0$ and $a_{1}-c^{2}>0$, (1.1) has four kink wave solutions $\pm u_{4}(x, t)$ and $\pm u_{5}(x, t)$.

\subsection{Remarks}

Remark 1. Some previous results becoming our special cases. For example, our results $u_{1}(x, t)$ and $u_{2}(x, t)$ become Li's results (see (4.10) and (4.9) in [7]) when $\delta=1, p=1$ and $\phi_{i}=0(i=1,3)$. Our results $\pm u_{2}(x, t)$ become Li's results (see (5.7) in [7]) when $\delta=1$ and $p=2$. When $\delta=1, p=1$ and $c=1$, Our results $u_{3}(x, t)$ 
becomes Li's results (see (4.7) in [7]). When $\delta=1, p=2$ and $c=1$, Our results $\pm u_{3}(x, t)$ become Li's results (see (5.2) in [7]). When $\delta=1, p=2, a_{2}=4 \sqrt{\frac{\left(a_{1}-c^{2}\right)}{3}}$ and $\omega=a_{2}$, Our results $\pm u_{4}(x, t)$ and $\pm u_{5}(x, t)$ become Li's results (see (5.9) and (5.10) in $[7])$.

Remark 2. Our some results obtained by using bifurcation method and Zhang's some results [1] obtained by using the judgement methods and solution formulae are the same, i.e., Zhang's results (1.6) and (1.7) are the same with our results (1.13) and (1.14). When $\omega=a_{2}$ and $c^{2}=a_{1}-\frac{a_{2}^{2}(p+1)}{a_{3}(p+2)^{2}}$, our results $u_{4}(x, t)$ and $u_{5}(x, t)$ become Zhang's result (1.8).

Remark 3. Our results include Zhang's results[8], i.e., when $p$ is even, $\delta=1, a_{3}<$ $0, a_{2}>0$ and $\frac{a_{2}^{2}}{4 a_{3}}<a_{1}-c^{2}<0$, our result $u_{2}(x, t)$ becomes Zhang's result (see (4.6) in [8]), and our results $\pm u_{4}(x, t)$ and $\pm u_{5}(x, t)$ become Zhang's result (see (4.19) and (4.20) in [8]).

\section{Preliminaries}

To derive our results, we given some preliminaries in this section. Substituting $u(x, t)=\varphi(\xi)$ with $\xi=x-c t$ ( $c$ is a constant) into (1.1), we have the following ordinary differential equation

$$
c^{2} \varphi^{\prime \prime}-c^{2} \delta \varphi^{(4)}-\left(a_{1} \varphi+a_{2} \varphi^{p+1}+a_{3} \varphi^{2 p+1}\right)^{\prime \prime}=0 .
$$

Integrating (2.1) twice and letting integral constants be zero, we get

$$
c^{2} \varphi-c^{2} \delta \varphi^{\prime \prime}-\left(a_{1} \varphi+a_{2} \varphi^{p+1}+a_{3} \varphi^{2 p+1}\right)=0 .
$$

Via (2.2) we establish a planar system

$$
\frac{d \varphi}{d \xi}=y, \quad \frac{d y}{d \xi}=\frac{\left(c^{2}-a_{1}\right) \varphi-a_{2} \varphi^{p+1}-a_{3} \varphi^{2 p+1}}{c^{2} \delta} .
$$

Obviously, if let

$$
H(\varphi, y)=c^{2} \delta y^{2}+\varphi^{2}\left(\frac{a_{3}}{p+1} \varphi^{2 p}+\frac{2 a_{2}}{p+2} \varphi^{p}+a_{1}-c^{2}\right),
$$

then system (2.3) has first integral

$$
H(\varphi, y)=h,
$$

where $h$ is integral constant.

In the following, we discuss bifurcation phase portraits of system (2.3). 


\subsection{Bifurcation phase portraits when $p$ is odd}

Let

$$
f(\varphi)=\left(c^{2}-a_{1}\right) \varphi-a_{2} \varphi^{p+1}-a_{3} \varphi^{2 p+1},
$$

then system $(2.3)$ reduces to

$$
\frac{d \varphi}{d \xi}=y, \quad \frac{d y}{d \xi}=\frac{f(\varphi)}{c^{2} \delta} .
$$

Thus, to study the the distribution of singular points of system $(2.3)$, we need to investigate the zero points of $f(\varphi)$. If $p$ is odd, $a_{3} \neq 0$, and $a_{2}^{2}-4 a_{3}\left(a_{1}-c^{2}\right) \geq 0$, then we get three zero points of $f(\varphi)$ as

$$
\varphi_{0}=0, \varphi_{-}=\left(\frac{-a_{2}-\sqrt{a_{2}^{2}-4 a_{3}\left(a_{1}-c^{2}\right)}}{2 a_{3}}\right)^{\frac{1}{p}}, \varphi_{+}=\left(\frac{-a_{2}+\sqrt{a_{2}^{2}-4 a_{3}\left(a_{1}-c^{2}\right)}}{2 a_{3}}\right)^{\frac{1}{p}},
$$

and two zero points of $f^{\prime}(\varphi)$ as

$$
\begin{gathered}
\tilde{\varphi}_{1}=\left(\frac{-(p+1) a_{2}-\sqrt{(p+1)^{2} a_{2}^{2}-4 a_{3}(2 p+1)\left(a_{1}-c^{2}\right)}}{2 a_{3}(2 p+1)}\right)^{\frac{1}{p}}, \\
\tilde{\varphi}_{2}=\left(\frac{-(p+1) a_{2}+\sqrt{(p+1)^{2} a_{2}^{2}-4 a_{3}(2 p+1)\left(a_{1}-c^{2}\right)}}{2 a_{3}(2 p+1)}\right)^{\frac{1}{p}} .
\end{gathered}
$$

Suppose $\left(\varphi^{*}, 0\right)$ is a singular point of system $(2.7)$, then at $\left(\varphi^{*}, 0\right)$ the eigenvalue of the linearized system of system $(2.7)$ is

$$
\lambda_{ \pm}\left(\varphi^{*}, 0\right)= \pm \sqrt{\frac{f^{\prime}\left(\varphi^{*}\right)}{c^{2} \delta}} .
$$

According to the theory of dynamical systems (e.g.,[12,13]), we obtain the following conclusions.

(1) When $\delta f^{\prime}\left(\varphi^{*}\right)<0,\left(\varphi^{*}, 0\right)$ is a center point.

(2) When $\delta f^{\prime}\left(\varphi^{*}\right)>0,\left(\varphi^{*}, 0\right)$ is a saddle point.

(3) When $f^{\prime}\left(\varphi^{*}\right)=0,\left(\varphi^{*}, 0\right)$ is a degenerate saddle point.

Solving equations $f^{\prime}\left(\varphi^{*}\right)=0$ and $H\left(\varphi^{*}, 0\right)=0$ respectively, we get two bifurcation curves $L_{1}$ and $L_{2}$ as follows

$$
L_{1}: a_{3}=\frac{1}{4\left(a_{1}-c^{2}\right)} a_{2}^{2},
$$

and

$$
L_{2}: a_{3}=\frac{p+1}{\left(a_{1}-c^{2}\right)(p+2)^{2}} a_{2}^{2} .
$$


From the above analysis, we obtain the bifurcation phase portraits of system (2.3) as Fig.1(A), Fig.2(C), Fig.3(E), Fig.4(G), Fig.5(I) and Fig.6(K).

\subsection{Bifurcation phase portraits when $p$ is even}

When $p$ is even, similar to above analysis, we obtain the bifurcation phase portraits of system (2.3) as Fig.1(B), Fig.2(D), Fig.3(F), Fig.4(H), Fig.5(J) and Fig.6(L).

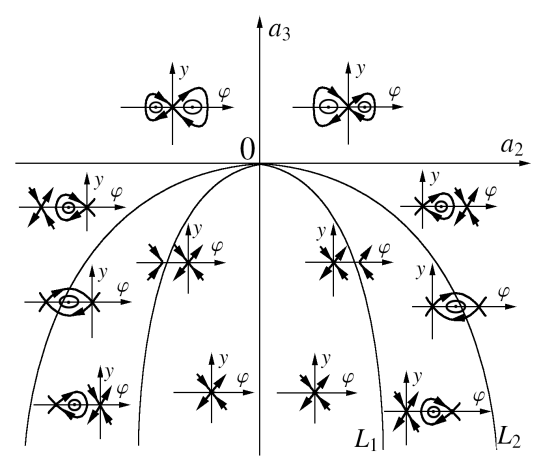

(A) $p$ is odd

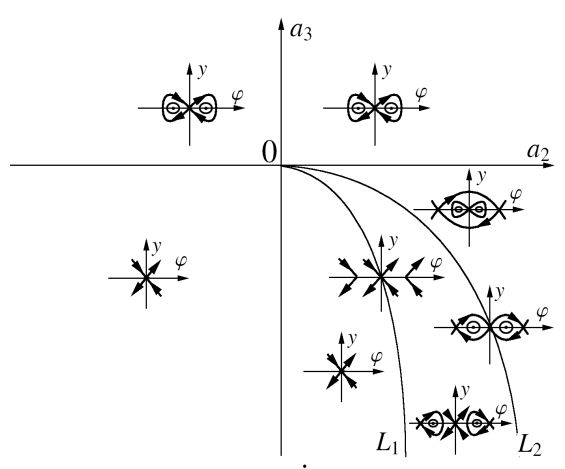

(B) $p$ is even

Figure 1: The bifurcation phase portraits of (2.3) when $\delta>0$ and $a_{1}-c^{2}<0$.

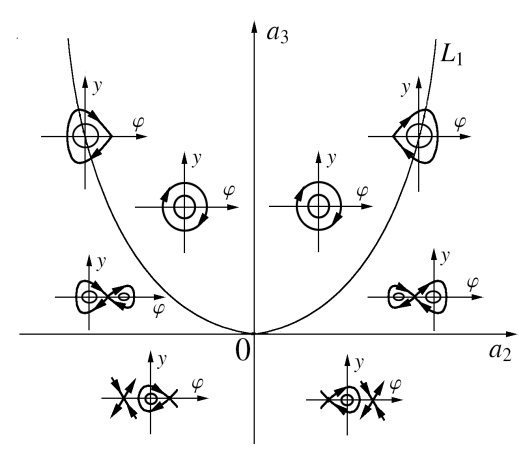

(C) $p$ is odd

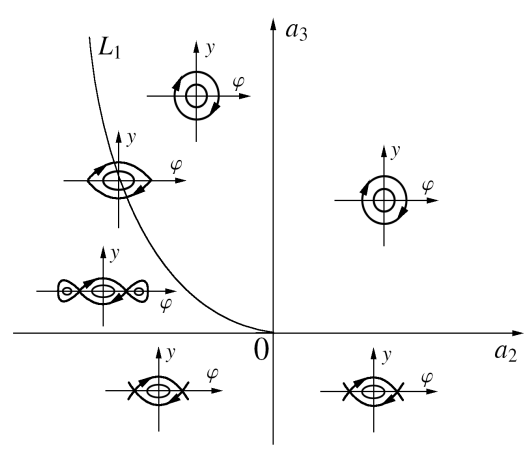

(D) $p$ is even

Figure 2: The bifurcation phase portraits of (2.3) when $\delta>0$ and $a_{1}-c^{2}>0$.

\section{Derive of Main Results}

In this section, we derive the Theorem $i(i=1,2,3,4)$. Firstly, we derive the Theorem 1. 


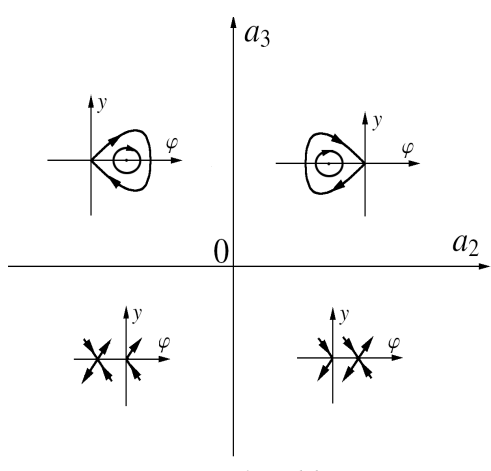

(E) $p$ is odd

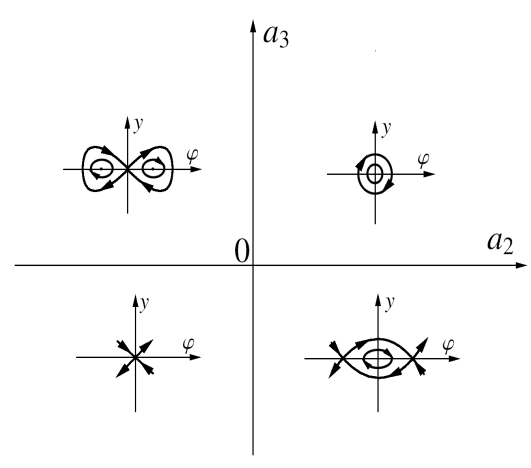

(F) $p$ is even

Figure 3: The bifurcation phase portraits of (2.3) when $\delta>0$ and $a_{1}-c^{2}=0$.

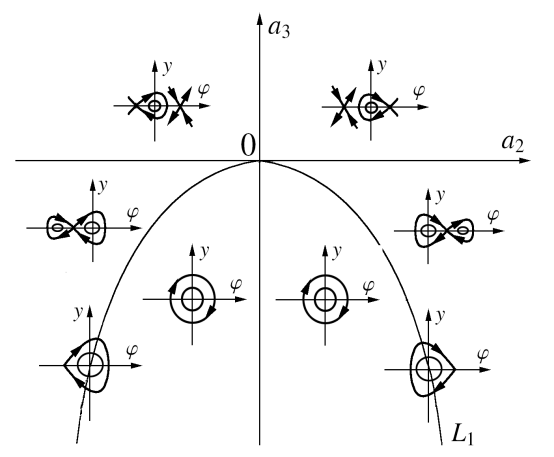

(G) $p$ is odd

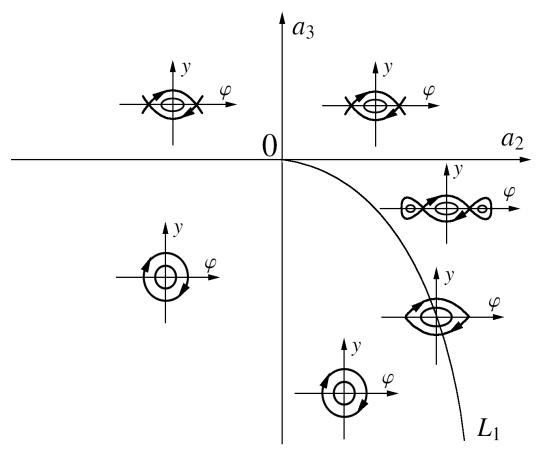

( $H$ ) $p$ is even

Figure 4: The bifurcation phase portraits of (2.3) when $\delta<0$ and $a_{1}-c^{2}<0$.

(1) When $p$ is odd, $\delta>0, a_{2} \neq 0, a_{3}>0$ and $a_{1}-c^{2}<0$, from Fig. 1(A) we see that system (2.3) has two homoclinic orbits (denote them as $\Gamma_{1}$ and $\Gamma_{2}\left(\right.$ or $\Gamma_{1}^{*}$ and $\left.\Gamma_{2}^{*}\right)($ see Fig.7)) which connect with singular point $(0,0)$.

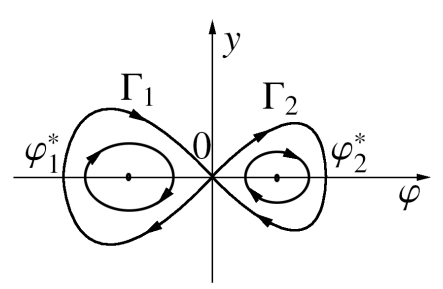

$a_{2}>0$

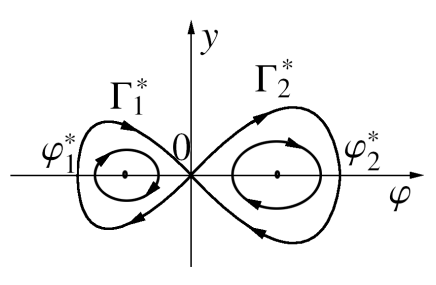

$a_{2}<0$

Fig. 7. Homoclinic orbits $\Gamma_{i}$ and $\Gamma_{i}^{*}(i=1,2)$ when $\delta>0, a_{3}>0$ and $a_{1}-c^{2}<0$. 


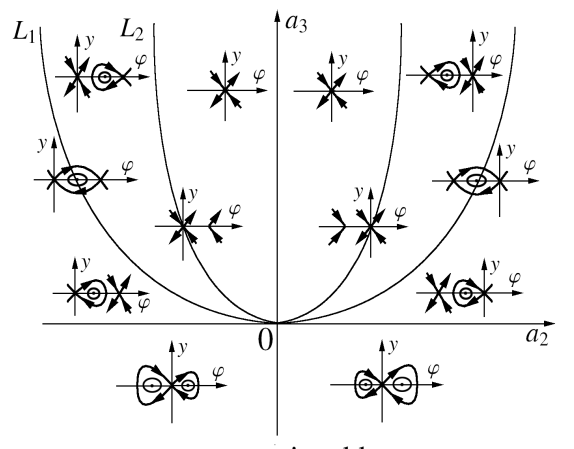

(I) $p$ is odd

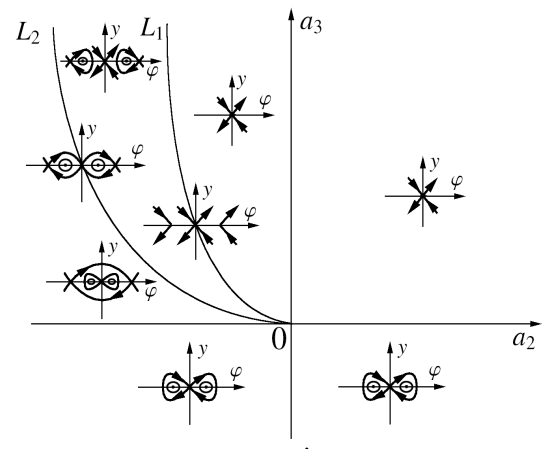

(J) $p$ is even

Figure 5: The bifurcation phase portraits of (2.3) when $\delta<0$ and $a_{1}-c^{2}>0$.

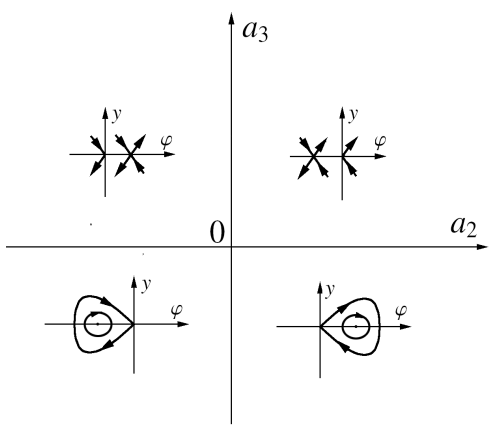

(K) $p$ is odd

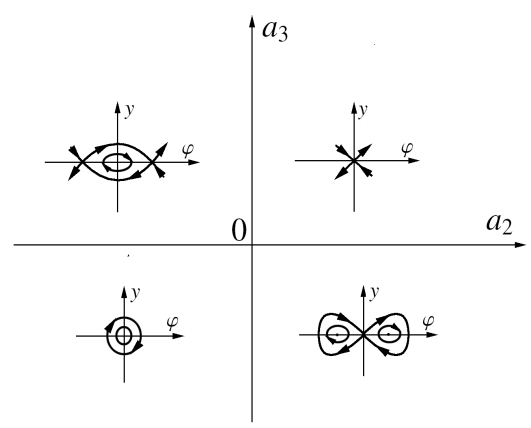

(L) $p$ is even

Figure 6: The bifurcation phase portraits of (2.3) when $\delta<0$ and $a_{1}-c^{2}=0$.

On $\varphi$-y plane, $\Gamma_{1}$ and $\Gamma_{2}$ (or $\Gamma_{1}^{*}$ and $\Gamma_{2}^{*}$ ) are expressed by

$$
y^{2}=\frac{\varphi^{2}\left(c^{2}-a_{1}-\frac{2 a_{2}}{p+2} \varphi^{p}-\frac{a_{3}}{p+2} \varphi^{2 p}\right)}{c^{2} \delta}, \quad \varphi \in\left[\varphi_{1}^{*}, \varphi_{2}^{*}\right],
$$

where

$$
\begin{aligned}
& \varphi_{1}^{*}=\left(\frac{-(p+1) a_{2}}{(p+2) a_{3}}-\frac{p+1}{a_{3}} \sqrt{\frac{a_{2}^{2}}{(p+2)^{2}}+\frac{a_{3}\left(c^{2}-a_{1}\right)}{p+1}}\right)^{\frac{1}{p}}, \\
& \varphi_{2}^{*}=\left(\frac{-(p+1) a_{2}}{(p+2) a_{3}}+\frac{p+1}{a_{3}} \sqrt{\frac{a_{2}^{2}}{(p+2)^{2}}+\frac{a_{3}\left(c^{2}-a_{1}\right)}{p+1}}\right)^{\frac{1}{p}} .
\end{aligned}
$$


Substituting (3.1) into $\frac{d \varphi}{d \xi}=y$, we have

$$
\pm \sqrt{\frac{c^{2} \delta}{\varphi^{2}\left(c^{2}-a_{1}-\frac{2 a_{2}}{p+2} \varphi^{p}-\frac{a_{3}}{p+2} \varphi^{2 p}\right)}} d \varphi=d \xi .
$$

Assuming $\varphi(0)=\varphi_{1}^{*}$ and integrating (3.4) along homoclinic orbit $\Gamma_{1}$, we get

$$
\int_{\varphi_{1}^{*}}^{\varphi} \frac{|c| \sqrt{\delta}}{s \sqrt{c^{2}-a_{1}-\frac{2 a_{2}}{p+2} s^{p}-\frac{a_{3}}{p+1} s^{2 p}}} d s=\int_{\xi}^{0} d s, \quad \xi>0
$$

and

$$
-\int_{\varphi}^{\varphi_{1}^{*}} \frac{|c| \sqrt{\delta}}{s \sqrt{c^{2}-a_{1}-\frac{2 a_{2}}{p+2} s^{p}-\frac{a_{3}}{p+1} s^{2 p}}} d s=\int_{0}^{\xi} d s, \quad \xi \leq 0 .
$$

Completing above two integrals we obtain

$$
\varphi(\xi)=\left(\frac{(p+2)\left(a_{1}-c^{2}\right)}{\alpha \cosh \beta \xi-a_{2}}\right)^{\frac{1}{p}}
$$

where $\alpha$ and $\beta$ are in (1.15). From $u(x, t)=\varphi(\xi)$ and $\xi=x-c t$, we obtain $u_{1}(x, t)$ as formula (1.13). Similarly, assuming $\varphi(0)=\varphi_{2}^{*}$ and integrating (3.4) along homoclinic orbit $\Gamma_{2}$, we get

$$
\int_{\varphi}^{\varphi_{2}^{*}} \frac{|c| \sqrt{\delta}}{s \sqrt{c^{2}-a_{1}-\frac{2 a_{2}}{p+2} s^{p}-\frac{a_{3}}{p+1} s^{2 p}}} d s=\int_{0}^{\xi} d s, \quad \xi<0
$$

and

$$
-\int_{\varphi_{2}^{*}}^{\varphi} \frac{|c| \sqrt{\delta}}{s \sqrt{c^{2}-a_{1}-\frac{2 a_{2}}{p+2} s^{p}-\frac{a_{3}}{p+1} s^{2 p}}} d s=\int_{\xi}^{0} d s, \quad \xi \geq 0 .
$$

Completing above two integrals we obtain

$$
\varphi(\xi)=\left(\frac{(p+2)\left(c^{2}-a_{1}\right)}{\alpha \cosh \beta \xi+a_{2}}\right)^{\frac{1}{p}}
$$

where $\alpha, \beta$ are in (1.15). From (3.10) we obtain $u_{2}(x, t)$ as formula (1.14). Similarly, we can derive $(2),(3)$ of Theorem 1 and Theorem 2 .

Secondly, we derive the Theorem 3. From Fig. 1(A) we see that when $p$ is odd, $\delta>0, a_{2} \neq 0, a_{3}=a_{3}^{*}$ and $a_{1}-c^{2}<0$, system (2.3) has two heteroclonic orbits (denote them as $\Gamma_{i}(i=3,4)\left(\right.$ or $\left.\Gamma_{i}^{*}(i=3,4)\right)$ (see Fig. 8)) connecting with singular point $(0,0)$. 


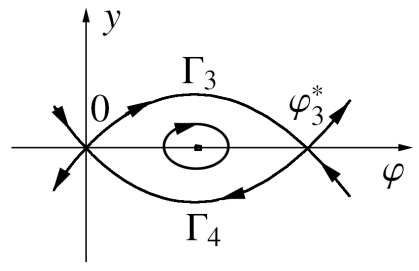

$a_{2}>0$

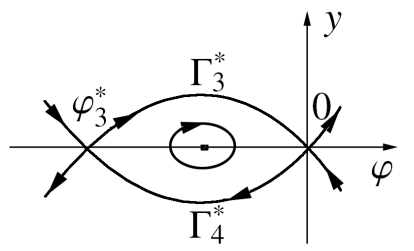

$a_{2}<0$

Fig. 8. Heteroclinic orbits $\Gamma_{i}$ and $\Gamma_{i}^{*}(i=3,4)$ When $\delta>0, a_{3}=a_{3}^{*}$ and $a_{1}-c^{2}<0$.

On $\varphi-y$ plane, $\Gamma_{3}$ and $\Gamma_{4}$ are expressed by

$$
y^{2}=\frac{\varphi^{2}}{c^{2} \delta}\left(c^{2}-a_{1}-\frac{2 a_{2}}{p+2} \varphi^{p}+\frac{a_{2}^{2}}{(p+2)^{2}\left(c^{2}-a_{1}\right)} \varphi^{2 p}\right), \quad \varphi \in\left(0, \varphi_{3}^{*}\right),
$$

$\Gamma_{3}^{*}$ and $\Gamma_{4}^{*}$ are expressed by

$$
y^{2}=\frac{\varphi^{2}}{c^{2} \delta}\left(c^{2}-a_{1}-\frac{2 a_{2}}{p+2} \varphi^{p}+\frac{a_{2}^{2}}{(p+2)^{2}\left(c^{2}-a_{1}\right)} \varphi^{2 p}\right), \quad \varphi \in\left(\varphi_{3}^{*}, 0\right),
$$

where

$$
\varphi_{3}^{*}=\left(\frac{(p+2)\left(c^{2}-a_{1}\right)}{a_{2}}\right)^{\frac{1}{p}} .
$$

Substituting (3.11) into $\frac{d \varphi}{d y}=y$ and integrating it along $\Gamma_{3}$ and $\Gamma_{4}$ respectively, we have

$$
\int_{\overline{\varphi_{0}}}^{\varphi} \frac{d s}{s \sqrt{c^{2}-a_{1}-\frac{2 a_{2}}{p+2} s^{p}-\frac{a_{2}^{2}}{(p+2)^{2}\left(c^{2}-a_{1}\right)} s^{2 p}}}=\int_{0}^{\xi} \frac{d s}{|c| \sqrt{\delta}}
$$

and

$$
-\int_{\overline{\varphi_{0}}}^{\varphi} \frac{d s}{s \sqrt{c^{2}-a_{1}-\frac{2 a_{2}}{p+2} s^{p}-\frac{a_{2}^{2}}{(p+2)^{2}\left(c^{2}-a_{1}\right)} s^{2 p}}}=\int_{0}^{\xi} \frac{d s}{|c| \sqrt{\delta}},
$$

where $\varphi(0)=\overline{\varphi_{0}} \in\left(0, \varphi_{3}^{*}\right)$ is a constant. Solving (3.14) it follows that

$$
\varphi(\xi)=\left(\frac{2 \sqrt{c^{2}-a_{1}}}{\nu e^{-\beta \xi}-2 \theta}\right)^{\frac{1}{p}} .
$$

Solving (3.15) we have

$$
\varphi(\xi)=\left(\frac{2 \sqrt{c^{2}-a_{1}}}{\nu e^{\beta \xi}-2 \theta}\right)^{\frac{1}{p}} .
$$


In (3.16) and (3.17) $\nu$ is a integral constant, $\beta$ is in (1.15) and

$$
\theta=-\frac{a_{2}}{(p+2) \sqrt{c^{2}-a_{1}}} .
$$

From (3.16), (3.17) we obtain $u_{4}(x, t)$ and $u_{5}(x, t)$ as (1.18) and (1.19).

Similarly, we can derive the Theorem 4 . Substituting $u_{i}(i=1,2,3,4,5)$ into (1.1), we find that they are its solutions indeed.

\section{Conclusions}

In this paper, we have employed bifurcation method in dynamical systems to study bifurcation phase portraits of (1.1)(see Fig.1-Fig.6). By using bifurcation phase portraits, its solitary wave solutions (see expressions (1.13), (1.14), (1.16)) and kink wave solutions (see expressions (1.18) and (1.19)) have been obtained.

Acknowledgment. Research is supported by the Natural Science Foundation of Guangdong Province (No 07006552) and the Department of Science and Technology of Yuxi City.

\section{References}

[1] W. G. Zhang, Q. S. Chang, E. G. Fan, Methods of judging shape of solitary wave and solution formulae for some evolution equations with nonlinear terms of high order, J. Math. Anal. Appl., 287(2003), 1-18.

[2] D. Kaya, The exact and numerical solitary-wave solutions for generalized modified Boussinesq equation, Phys. Lett., 348(2006)244-250.

[3] P. A. Clarkson, R. J. Leveque, R. Saxton, Solitary wave interactions in elastic rods, Stud. Appl. Math., 75(1986), 95-122.

[4] I. L. Bogolubsky, Some examples of inelastic solition interaction, Comput. Phys. Commun., 13(1977), 149-155.

[5] A. Parker, On exact solutions of the regularied long-wave equation: A direct approach to partially integrable equation, 1. solitary wave and solutions, J. Math. Phys., 36(1995), 3498-3505.

[6] W. G. Zhang, W. X. Ma, Explicit solitary-wave solutions to generalized PochhammerChree equations, Appl. Math. Mech., 20(1999), 666-674.

[7] J. B. Li, L. J. Zhang, Bifurcations of traveling wave solutions in generalized Pochhammer-Chree equation, Chaos Solitons Fractals, 14(2002), 581-593.

[8] W. L. Zhang, Solitary wave solutions and kink wave solutions for a generalized PC equation, Acta. Math. Appl. Sinica, English Series 21(2005), 125-134.

[9] M. Rafei, D. D. Ganji, H. R. Mohammadi Daniali, H. Pashaei, Application of homotopy perturbation method to the RLM and generalized modified Boussinesq equations, Phys. Lett., 364(2007), 1-6. 
[10] Z. R. Liu, Z. Y. Ouyang, A note on solitary wave for modified forms of Camassa-Holm and Degasperis-procesi equation, Phys. Lett., 366(2007), 377-381.

[11] T. Jiang, Z. Y. Yang, Z. R. Liu, Bounded traveling wave soultion of a nolinear equation, Kyungpook Math J., 43(2003), 113-125

[12] S. N. Chow, J. K. Hale, Method of bifurcation theory, Springer-verlag, New York, 1982.

[13] J. Guckenheimer, P. Homes, Nonlinear Oscillations, Dynamical Systems and Bifurcations of Vector Fields,Springer-verlag, New York, 1999. 